\title{
Unitarized Chiral Dynamics in Three-body systems
}

\author{
A. Martínez Torres* \\ Departamento de Física Teórica and IFIC, Centro Mixto Universidad deValencia-CSIC, \\ Institutos de Investigación de Paterna, Aptdo. 22085, 46071 Valencia, Spain. \\ E-mail: amartinedific.uv.es

\section{K. P. Khemchandani} \\ Centro de Física Teórica, Departamento de Física, Universidade de Coimbra, P-3004-516 \\ Coimbra, Portugal. \\ E-mail: kanchandteor.fis.uc.pt

\section{E. Oset} \\ Departamento de Física Teórica and IFIC, Centro Mixto Universidad deValencia-CSIC, \\ Institutos de Investigación de Paterna, Aptdo. 22085, 46071 Valencia, Spain. \\ E-mail: oset@ific.uv.es
}

\begin{abstract}
We have used unitary chiral dynamics in a coupled channel approach to solve the Faddeev equations for different two meson-one baryon and three meson systems. We have found many resonances in the strangeness $S=0,-1$ and 1 sector which can be identified with many of the $1 / 2^{+}$ states listed by the Particle Data Group (PDG). In concrete, in the strangeness $S=0$ sector, we have studied the $\pi \pi N$ system and its coupled channels and we report three $N^{*}$ states, $N^{*}(1710)$, $N^{*}(1920), N^{*}(2100)$ and one $\Delta$ resonance, $\Delta(1910)$, with $J^{P}=1 / 2^{+}$. The state at $1920 \mathrm{MeV}$ is not listed by the PDG and it was predicted by the authors of [1]. In the strangeness $S=-1$ sector, we have solved the equations for the $\pi \bar{K} N$ system and its coupled channels and we find four $\Sigma$ 's and two $\Lambda$ 's, in the $1500-1800 \mathrm{MeV}$ region, as two meson-one baryon $\mathrm{S}$-wave $1 / 2^{+}$resonances, which correspond to all the low-lying $S=-1$ and $J^{P}=1 / 2^{+}$states listed by the PDG. We have investigated the possibility to generate dynamically states with $S=1$ in the $\pi K N$ system and we have observed a broad structure around $1700 \mathrm{MeV}$ with a width of $200 \mathrm{MeV}$ which could be observed in reactions involving the $\pi K N$ channel in the final state interaction. We have also studied three meson systems like $\phi K \bar{K}, \phi \pi \pi$ and $J / \psi K \bar{K}, J / \psi \pi \pi$ and found, in the first one, dynamical generation of a resonance with $\phi f_{0}(980)$ structure, $J^{P C}=1^{--}$and a mass around $2150 \mathrm{MeV}$, which can be identified with the $X(2175)$ resonance and, for the second one, a peak close to 4200 $\mathrm{MeV}$ which can be identified with the $Y(4260)$ state observed by different experimental groups
\end{abstract}

6th International Workshop on Chiral Dynamics, CD09

July 6-10, 2009

Bern, Switzerland

\footnotetext{
*Speaker.
} 


\section{Introduction}

During the last twenty years non-perturbative approaches based on chiral Lagrangians and unitarity in coupled channels have been a very important tool in order to reproduce and understand many properties of the low-lying $1 / 2^{-}$baryon resonances as well as the $0^{+}$states in the meson sector. For example, the study with this technique of different two-body meson-baryon and mesonmeson systems in $S$-wave, like $\bar{K} N, \pi \Sigma, \pi \Lambda, \pi N, \pi \pi, K \bar{K}, \pi \eta$, etc., has led to the dynamical generation of the $\Lambda(1405), \Lambda(1520), N^{*}(1535)$, etc., in the baryon sector and the $f_{0}(980), a_{0}(980)$, $\sigma(600)$, etc., in the meson sector $[2,3,4,5,6,7,8,9,10]$. However, while the properties of many baryon states in the $J^{P}=1 / 2^{-}$sector are well understood within the unitary chiral models, the $J^{P}=1 / 2^{+}$domain remains far less understood, both experimentally and theoretically. The situation becomes worse for the $1 / 2^{+}$hyperon resonances as compared to the $1 / 2^{+}$nucleon ones.

The good reproduction of the low lying $1 / 2^{-}$and $0^{+}$states in the $S$-wave meson-baryon and meson-meson interaction, respectively, using unitary chiral dynamics and the strongly attractive interaction in these two-body systems, suggests that the addition of a pseudoscalar meson in Swave could lead to a description of the $1 / 2^{+}$resonances. There exist many findings which hint towards this possibility. For example, the $\Sigma(1660)$ state shows up as a clear signal in the reaction $K^{-} p \rightarrow \pi^{0} \pi^{0} \Sigma^{0}[11]$, the $N^{*}(1710)$ decays with $40-90 \%$ branching ratio to $\pi \pi N$ and with $10-40 \%$ for that $\pi \pi N$ system in which the $\pi-\pi$ interaction is in s wave, with isospin zero [12], etc.

Recently, the existence of a new meson resonance, $X(2175)$, has been claimed by BABAR and BES $[13,14,15]$ in the $e^{+} e^{-} \rightarrow \phi f_{0}(980)$ and $J / \psi \rightarrow \eta \phi f_{0}(980)$ reactions with quantum numbers $1^{--}$. Further, an enhancement in the data for the $\pi^{+} \pi^{-} J / \psi$ invariant mass spectrum was found near $4.26 \mathrm{GeV}$ by several experimental groups $[16,17,18,19]$, indicating the presence of a resonance which was named as $Y(4260)$ and it was found to be of $J^{P C}=1^{--}$nature. Since in the chiral models the $f_{0}(980)$ gets dynamically generated in the $K \bar{K}$ and $\pi \pi$ interaction [4, 8], there is a possibility to explain the $X(2175)$ and the $Y(4260)$ states as resonances in the $\phi K \bar{K}$ and $J / \psi K \bar{K}$ systems, respectively, and its coupled channels.

All these findings demand, thus, a study of two meson and one baryon systems, like $\pi \pi N$, $\pi \pi \Sigma, \pi \bar{K} N$, etc., and three-meson systems like, $\phi \pi \pi, \phi K \bar{K}, J / \psi K \bar{K}, J / \psi \pi \pi$, etc.,

\section{Formalism}

Our objective is to calculate the three-body $T$-matrix which describes the interaction between the different particles and search for peaks in the squared amplitude which could be identified with physical states. In order to get the three-body $T$ matrix we follow the procedure developed in $[20,21,22,23,24]$. One of the important features of this approach is an explicit cancellation between the contribution of the off-shell part of the chiral $t$-matrices to the Faddeev equations with a three-body contact term originating from the chiral Lagrangian, which renders the integral Faddeev equations into the following algebraic ones

$$
\begin{aligned}
& T_{R}^{12}=t^{1} g^{12} t^{2}+t^{1}\left[G^{121} T_{R}^{21}+G^{123} T_{R}^{23}\right] \\
& T_{R}^{13}=t^{1} g^{13} t^{3}+t^{1}\left[G^{131} T_{R}^{31}+G^{132} T_{R}^{32}\right]
\end{aligned}
$$




$$
\begin{aligned}
& T_{R}^{21}=t^{2} g^{21} t^{1}+t^{2}\left[G^{212} T_{R}^{12}+G^{213} T_{R}^{13}\right] \\
& T_{R}^{23}=t^{2} g^{23} t^{3}+t^{2}\left[G^{231} T_{R}^{31}+G^{232} T_{R}^{32}\right] \\
& T_{R}^{31}=t^{3} g^{31} t^{1}+t^{3}\left[G^{312} T_{R}^{12}+G^{313} T_{R}^{13}\right] \\
& T_{R}^{32}=t^{3} g^{32} t^{2}+t^{3}\left[G^{321} T_{R}^{21}+G^{323} T_{R}^{23}\right]
\end{aligned}
$$

The $T_{R}^{i j}$ partitions include all the different contributions to the three-body $T$ - matrix in which the last interactions are given in terms of the two-body $t$-matrices $t^{j}$ and $t^{i}$, respectively. The $T_{R}^{i j}$ matrices are connected to the Faddeev partitions $T^{i}$ through

$$
T^{i}=t^{i} \delta^{3}\left(\vec{k}_{i}^{\prime}-\vec{k}_{i}\right)+\sum_{j \neq i=1}^{3} T_{R}^{i j}, \quad i=1,2,3
$$

with $\vec{k}_{i}\left(\vec{k}_{i}^{\prime}\right)$ being the initial (final) momentum of the particle $i$. Therefore, the full three-body $T$-matrix is expressed in terms of $T_{R}^{i j}$ as

$$
\begin{aligned}
T & =\sum_{i=1}^{3} T^{i}=\sum_{i=1}^{3} t^{i} \delta^{3}\left(\vec{k}_{i}^{\prime}-\vec{k}_{i}\right)+T_{R} \\
T_{R} & \equiv \sum_{i=1}^{3} \sum_{j \neq i=1}^{3} T_{R}^{i j}
\end{aligned}
$$

In Eq. (2.1), $g^{i j}$ is the three-body Green's function of the system and $G^{i j k}$ is a loop function of three-particles (for more details see [20, 21, 22, 23, 24]). All the matrices involved in Eq. (2.1) are projected in $S$-wave, thus giving total $J^{\pi}=1 / 2^{+}$.

\section{Results}

\subsection{Two pseudoscalar mesons and one baryon with $S=0$}

We solve Eqs. (2.1) for the $\pi \pi N$ system and coupled channels, i.e, $\pi \pi N, \pi K \Sigma, \pi K \Lambda$ and $\pi \eta N$, taking into account fourteen coupled channels for total charge zero [22, 23]. The calculated three-body $T$-matrix reveals dynamical generation of four resonances which can be identified with several $1 / 2^{+} N^{*}$ states listed by the PDG:

1. $N^{*}(1710)$ : we obtain a peak with mass around $1704 \mathrm{MeV}$ and $375 \mathrm{MeV}$ of width, when the two pions are resonating close to the $\sigma(600)$ region, which we identify with the $N^{*}(1710)$. This finding of the $\sigma(600) N$ structure gives a natural interpretation for the large width of the $N^{*}(1710)$.

2. $N^{*}(2100)$ : another peak with mass around that of the $N^{*}(2100)$, with one of the $\pi N$ pairs simultaneously resonating as the $N^{*}(1650)$, implying a $\pi N^{*}(1650)$ structure, is found after solving Eqs. (2.1) .

3. $\Delta(1910)$ : we observe a peak in the $\pi K \Lambda$ channel whose properties correspond to the $\Delta(1910)$. 
4. $N^{*}(1920)$ ?: a peak around $1920 \mathrm{MeV}$ shows up in the $T$-matrix for the $K \bar{K} N$ channel with total isospin $1 / 2, J^{\pi}=1 / 2^{+}$, when the $K \bar{K}$ system clusters to generate the $f_{0}(980)$ and the $a_{0}(980)$ resonances. This state is not listed by the PDG and it was already suggested by the authors of [1]. In fact, there are some experimental findings which favor the existence of a $N^{*}$ with spin-parity $1 / 2^{+}$at an energy close to $1910 \mathrm{MeV}$, although better experimental studies are needed to confirm this state (see more arguments in [25]).

\subsection{Two pseudoscalar mesons and one baryon system with $S=-1$}

It is well known that the $\Lambda(1405) S_{01}$ resonance gets dynamically generated in the $\pi \Sigma$ system and its coupled channels $[2,7]$. Since the interaction in the $\pi \Sigma$ region is attractive and the two pion interaction is also strongly attractive in nature around the $\sigma(600)$ and $f_{0}(980)$ mass $[4,8]$, the addition of one more pion to the $\pi \Sigma$ system will result into a system in which the interaction between the different pairs is attractive in some energy range. This means that systems as $\pi \pi \Sigma$, $\pi \pi \Lambda$, etc., can get bound through the mutual interaction between the three particles. We have solved Eqs. (2.1) for the $\pi \pi \Sigma$ system and coupled channels considering twenty-two coupled channels for total charge of the three particles equal to zero [20]. As result, all the low-lying $1 / 2^{+} \Sigma$ and $\Lambda$ states known so far along with some controversial states, i.e, $\Sigma(1770), \Sigma(1660), \Sigma(1620), \Sigma(1560)$, $\Lambda(1810)$ and $\Lambda(1600)$, get dynamically generated as two meson-one baryon resonances.

\subsection{Baryon $S=1$ resonances in the $N \pi K$ system}

Since the $K N$ interaction obtained from chiral Lagrangians is repulsive in nature [26], it is appealing to think that the addition of a pion, which acts like a "glue" between the $K$ and the $N$ since the $\pi N$ and $K N$ interactions are attractive in some energy range [3, 4, 8], could lead to a bound state around a mass close to $m_{\pi}+m_{K}+m_{N}=1540 \mathrm{MeV}$. This is exactly the energy region in which a peak in the $K^{+} n$ invariant mass for the $\gamma n \rightarrow K^{+} K^{-} n$ reaction was observed on a ${ }^{12} C$ target at Spring8/Osaka [27], raising great hopes that for the first time a strangeness $S=1$ narrow exotic baryon could be found, the $\Theta^{+}$. Some investigations of the $N \pi K$ system were already made and they weakly concluded the difficulty to get this system as a bound state [28, 29].

However our finding of several resonances in the corresponding $S=-1$ system motivated us to use the same formalism and calculate the amplitude for the $N \pi K$ system. In agreement with the previous works, we do not find any structure in the energy region close to $1542 \mathrm{MeV}$, but we get a bump around $1720 \mathrm{MeV}$ with about $200 \mathrm{MeV}$ of width and with isospin zero [24], which we expect to be studied experimentally in the future.

\subsection{Three meson systems with $S=0$}

In [30] a study of the $e^{+} e^{-} \rightarrow \phi f_{0}(980)$ reaction was made through a loop mechanism involving pseudoscalar and vector meson loops, and by keeping the final state as plane waves, which could explain the background observed by different experimental groups [13, 14, 15], but not the experimental peak obtained around an energy of $2175 \mathrm{MeV}$ in the invariant mass of $\phi f_{0}(980)[13,14,15]$. In the chiral models, the $f_{0}(980)$ resonance appears in the $K \bar{K}$ interaction with a strong coupling to that channel [4]. Therefore, a study of the $\phi K \bar{K}$ system could explain the experimental results. We solved Eqs. (2.1) taking as coupled channels $\phi K \bar{K}$ and $\phi \pi \pi$ with total charge zero and we obtain 
a peak around $2150 \mathrm{MeV}$ [21] with a width of $27 \mathrm{MeV}$ when the $K \bar{K}$ and $\pi \pi$ system is resonating close to the $f_{0}(980)$ region, thus, confirming the experimental finding. We implemented the result of [30] as the final state interaction for the $e^{+} e^{-} \rightarrow \phi f_{0}(980)$ and calculated the cross section for that process. The result is a peak in the cross section around $2175 \mathrm{MeV}$ which is in agreement with the experimental cross section obtained by different experimental groups $[13,14,15]$.

We have also investigated the possibility to generate dynamically a resonance in the $J / \psi K \bar{K}$, $J / \psi \pi \pi$ system. To do that, we have consider $J / \psi K \bar{K}$ and $J / \psi \pi \pi$ as coupled channels and we have solved Eqs. (2.1). As a result we get a peak close to $4200 \mathrm{MeV}$, when the $K \bar{K}$ system generate the $f_{0}(980)$ resonance, which can be identified with the $Y(4260)$ state observed by several experimental groups $[15,16,17,18,19]$

\section{Conclusions}

Using unitary chiral dynamics, we have developed a formalism where the integral Faddeev equations become algebraic ones and which can be solved by inverting matrices. We have used this formalism to investigate possible generation of resonances in the $\pi \pi N$ system and coupled channels, which resulted into dynamical generation of two $N^{*}$ 's and one $\Delta$ states, corresponding to the $N^{*}(1710), N^{*}(2100)$ and $\Delta(1910)$, respectively, and a new $N^{*}$ state with a mass close to 1920 $\mathrm{MeV}$ and $J^{P}=1 / 2^{+}$. We have also studied the $\pi \pi \Sigma$ system and its coupled channels and we have obtained the dynamical generation of four $\Sigma$ 's and two $\Lambda$ 's with $J^{P}=1 / 2^{+}$, which can be identified with the low lying $1 / 2^{+} \Sigma$ and $\Lambda$ states listed by the PDG in the energy region $1500-1800 \mathrm{MeV}$. We also investigated the $\pi K N$ system to search for possible $S=1$ states. In the meson sector, we have studied the $\phi K \bar{K}$ and $\phi \pi \pi$ systems getting the dynamical generation of the $X(2175)$ resonance, which was observed by several experimental groups, and the $J / \psi K \bar{K}, J / \psi \pi \pi$ system obtaining a peak around $4200 \mathrm{MeV}$ which can be identified with the $Y(4260)$ state.

\section{Acknowledgments}

This work is partly supported by DGICYT contract number FIS2006-03438. We acknowledge the support of the European Community-Research Infrastructure Integrating Activity "Study of Strongly Interacting Matter" (acronym HadronPhysics2, Grant Agreement n. 227431) under the Seventh Framework Programme of EU. K. P. Khemchandani thanks the support by the Fundação para a Ciência e a Tecnologia of the Ministério da Ciência, Tecnologia e Ensino Superior of Portugal (SFRH/BPD/40309/2007). A. M. T is supported by a FPU grant of the Ministerio de Ciencia e Innovación.

\section{References}

[1] D. Jido and Y. Kanada-En'yo, Phys. Rev. C 78, 035203 (2008).

[2] E. Oset and A. Ramos, Nucl. Phys. A 635, 99 (1998)

[3] T. Inoue, E. Oset and M. J. Vicente Vacas, Phys. Rev. C 65, 035204 (2002)

[4] J. A. Oller and E. Oset, Nucl. Phys. A 620, 438 (1997) [Erratum-ibid. A 652, 407 (1999)] 
[5] J. Nieves and E. Ruiz Arriola, Nucl. Phys. A 679, 57 (2000)

[6] B. Borasoy, R. Nissler and W. Weise, Eur. Phys. J. A 25, 79 (2005)

[7] D. Jido, J. A. Oller, E. Oset, A. Ramos and U. G. Meissner, Nucl. Phys. A 725, 181 (2003)

[8] J. A. Oller, E. Oset and J. R. Pelaez, Phys. Rev. Lett. 80, 3452 (1998).

[9] V. Bernard, N. Kaiser and U. G. Meissner, Phys. Rev. C 52, 2185 (1995).

[10] L. Roca, S. Sarkar, V. K. Magas and E. Oset, Phys. Rev. C 73, 045208 (2006).

[11] S. Prakhov et al. [Crystall Ball Collaboration], Phys. Rev. C 70, 034605 (2004).

[12] C. Amsler et al. (Particle Data Group), Physics Letters B667, 1 (2008) .

[13] B. Aubert et al. [BABAR Collaboration], Phys. Rev. D 74, 091103 (2006).

[14] B. Aubert et al. [BABAR Collaboration], Phys. Rev. D 76, 012008 (2007).

[15] M. Ablikim et al. [BES Collaboration], Phys. Rev. Lett. 100, 102003 (2008).

[16] B. Aubert et al. [BABAR Collaboration], Phys. Rev. Lett. 95 142001(2005).

[17] T. E. Coan et al. [CLEO Collaboration], Phys. Rev. Lett. 96, 162003 (2006).

[18] Q. He et al. [CLEO Collaboration], Phys. Rev. D 74, 091104 (2006).

[19] C. Z. Yuan et al. [Belle Collaboration], Phys. Rev. Lett. 99, 182004 (2007).

[20] A. Martinez Torres, K. P. Khemchandani and E. Oset, Phys. Rev. C 77, 042203 (2008).

[21] A. Martinez Torres, K. P. Khemchandani, L. S. Geng, M. Napsuciale and E. Oset, Phys. Rev. D 78, 074031 (2008).

[22] K. P. Khemchandani, A. Martinez Torres and E. Oset, Eur. Phys. J. A 37, 233 (2008).

[23] A. Martinez Torres, K. P. Khemchandani and E. Oset, arXiv:0812.2235 [nucl-th].

[24] K. P. Khemchandani, A. Martinez Torres and E. Oset, Phys. Lett. B 675, 407 (2009).

[25] A. Martinez Torres, K. P. Khemchandani, U. G. Meissner and E. Oset, Eur. Phys. J. A 41, 361 (2009) [arXiv:0902.3633 [nucl-th]].

[26] V. Bernard, N. Kaiser and U. G. Meissner, Int. J. Mod. Phys. E 4, 193 (1995) [arXiv:hep-ph/9501384].

[27] T. Nakano et al. [LEPS Collaboration], Phys. Rev. Lett. 91, 012002 (2003) [arXiv:hep-ex/0301020].

[28] P. Bicudo and G. M. Marques, Phys. Rev. D 69, 011503 (2004) [arXiv:hep-ph/0308073].

[29] F. J. Llanes-Estrada, E. Oset and V. Mateu, Phys. Rev. C 69, 055203 (2004) [arXiv:nucl-th/0311020].

[30] M. Napsuciale, E. Oset, K. Sasaki and C. A. Vaquera-Araujo, Phys. Rev. D 76, 074012 (2007). 